\title{
Caratteristiche idrodinamiche di alcuni laghi artificiali
}

\author{
(Hydrodinamical features of some artificial lakes)
}

\author{
L. Butera *
}

Ricevuto il 7 Aprile 1972

RIASSUNTO. - Dopo aver richiamato le equazioni utilizzate per lo studio delle sesse dei laghi, in questo lavoro sono stati studiati i modi di oscillazione di quattro bacini artificiali ubicati in Valle d'Aosta (Place Moulin, Beauregard), nel Cuneese (Piastra) e nel bacino idrico del fiume Adda (Alpe Gera).

Valutati i periodi col metodo di Hidaka, essi sono stati raffrontati con quelli desumibili da laghi di forma geometrica particolarmente semplice.

$\mathrm{Si}$ è infine eseguita l'integrazione numerica dell'equazione di Chrystal e si è provveduto al tracciamento delle ampiezze per le varie nodalità mediante un plotter direttamente collegato al calcolatore.

summar. - After reviewing the equations used for the study of the seiches of lakes, in this work the types of oscillation of four artificial lakes situated in the Aosta Valley (Place Moulin, Beauregard), in the Cuneo area (Piastra) and in the Adda river (Alpe Gera), reservoir liave been studied.

The periods valued with Hidaka's method have been compared with those obtained from lakes of very simple geometrical form.

Finally the numerical integration of Chrvstal's equation has been calculated and the amplitudes corresponding to the various nodes were traced by a plotter, directly linked to the calculator.

1. - Nel presente lavoro si è esaminato il comportamento di alcuni laghi artificiali nei riguardi delle oscillazioni proprie indotte da cause naturali di vario genere (vento, variazioni di pressione atmosferica, sismi ecc.).

Tali bacini artificiali presentano, rispetto ai laghi naturali l'indiscutibile vantaggio di una batimetria in genere assai ben conosciuta e la possibilità di un controllo sperimentale a diverse quote, cosa pra(Italia).

(*) Istituto di Idraulica e Costruzioni Idrauliche. Politecnico di Torino 
ticamente impossibile nei bacini naturali le cui escursioni di livello sono ridottissime rispetto alle profondità. In figura 2 sono rappresentate le curve normali di quattro laghi, oggetto di questo studio.

Ja un punto di vista teorico il problema puó essere affrontato ricorrendo ad uno schema di fluido perfetto; l'equazione del moto e di continuità permettono allora, unitamente alle condizioni ai limiti, di determinare i modi di oscillazione e l'andamento delle ampiezze, in alcuni casi di semplice forma geometrica del lago. Quando invece il bacino si presenta con andamento planimetrico e altimetrico particolarmente irregolare, occorre procedere in modo leggermente diverso come richiamato rapidamente al punto $2^{\circ}$ ) del paragrafo successivo.

2. - I'equazione del moto e l'equazione di continuità, nell'ambito dello schema indicato al numero precedente, si scrivono

$$
\begin{aligned}
& g \frac{i \eta}{\partial x}+\frac{\partial^{2} \xi}{\partial t^{2}}=0 \\
& b \eta+\frac{\partial(\Omega \xi)}{\partial x}=0
\end{aligned}
$$

da cui eliminando $\xi$ o $\eta$ si ricava

$$
\begin{gathered}
\left.\frac{\partial^{2} \xi}{\partial t^{2}}=g \frac{\partial}{\partial x} \mid \frac{1}{b}-\frac{\partial}{\partial x}(\Omega \xi)\right] \\
b \frac{\partial^{2} \eta}{\partial t^{2}}=g \frac{\partial}{\partial x}\left|\Omega \frac{\partial \eta}{\partial x}\right| .
\end{gathered}
$$

Se ad esempio il lago è di sezione trasversale rettangolare e $b$ è costante, dalla [3] si ottiene

$$
\frac{\partial^{2} \xi}{\partial t^{2}}=g-\frac{\partial^{2}}{\partial x^{2}}(h \xi)
$$

\begin{tabular}{|c|c|c|c|}
\hline$A$ & superficie del lago & $s(x)$ & superficie parziale del lago fino \\
\hline$a$ & lunghezza del lago & & all'ascissa $x$ \\
\hline b & larghezza del lago in superficie & $\sigma$ & curva normale del lago \\
\hline b & $\begin{array}{l}\text { profondita massima di una se. } \\
\text { zione (in quiete) }\end{array}$ & & $\begin{array}{l}\text { spostamento orizzontale } \\
\text { spostamento verticale }\end{array}$ \\
\hline & $\begin{array}{l}\text { sezione trasversale del lago (in } \\
\text { quiete) }\end{array}$ & $n, T$ & frequenza e periodo \\
\hline
\end{tabular}

cioe

$$
\frac{\partial^{2}(h \xi)}{\partial t^{2}}=g n \frac{\partial^{2}(h \xi)}{\partial x^{2}} .
$$

Elenco dei simboli 
Ricerchiamo una soluzione ponendo

$$
h \xi=P(x) \sin 2 \pi n t .
$$

Allora $P(x)$ deve soddisfare all'equazione

$$
\frac{d^{2} P}{d x^{2}}+\frac{4 \pi^{2} n^{2}}{g h} P=0
$$

Se ad esempio è (fig. 1)

$$
h=h_{0} \frac{x}{a}
$$

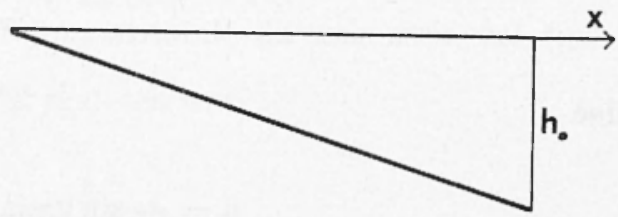

a

Fig. I - Lago con profondità linearmente variabile.

l'equazione [5] diviene

$$
\frac{d^{2} P}{d x^{2}}+\frac{4 \pi^{2} n^{2} a}{g h_{0} x} P=0
$$

e se si pone ancora $4 \pi^{2} n^{2} a / g h_{0}=K^{2}$, l'integrale generale della precedente si scrive:

$$
P=\sqrt{x}\left[A J_{1}(2 \pi \sqrt{x})+B Y_{1}(2 \pi \sqrt{x})\right]
$$

avendo indicato con $J$ e $Y$ le funzioni di Bessel di prima e seconda specie.

Poiché $\xi$, in genere non nullo, non è comunque mai $\infty$, è certo $B=0$, per cui la [6] si riduce a

$$
P=A \sqrt{x} J_{1}(2 \pi \sqrt{x}) .
$$

Se il lago termina con una parete verticale (ciò̀ praticamente col parametro di monte di una diga a gravità), risulta

$$
h \xi=0 \quad \text { per } x=a
$$


ed è possibile allora ricavare le frequenze di oscillazione corrispondenti alle diverse nodalità. Si ha:

$$
2 \pi \sqrt{a}=\varrho_{11}
$$

avendo indicato con $\varrho_{11}$ gli zeri della funzione $J_{1}$ e cioè $\varrho_{11}=3,832$; $\varrho_{12}=7,016 \ldots$ ecc.

Daila [2], per il caso considerato, cioè $\Omega=b h$, con $b=$ cost, si ricava

$$
\eta=-\frac{\partial(h \xi)}{\partial x}
$$

ciò̀

$$
\eta=-\sin 2 \pi n t-\frac{d P}{a x}
$$

La derivata che compare a secondo membro vale manifestamente (a meno di una costante) $J_{0}(2 K \sqrt{x})$. Quindi la posizione delle linee nodali corrisponde a

$$
2 K \sqrt{x}=\varrho_{01}
$$

con $K$ determinato dalla [7] e in cui $\underline{o 0}_{0 i}$ rappresenta gli zeri della funzione $J_{0}$.

In modo analogo si può procedere per bacini di sezione rettangolare, con profondità costante e larghezza linearmente variabile da 0 a $b_{0}$ operando sulla [4].

In questo caso la relazione che permette di determinare i periodi è

$$
T_{i}=\frac{2 \pi a}{\sqrt{g h_{0}} \varrho_{1 i}} .
$$

Anche in questo caso i periodi stanno nei rapporti degli zeri della funzione $J_{1}$.

Ma è facile comprendere che affrontare per questa via il problema di determinare le sesse in un lago naturale è praticamente impossibile.

Si riprenda allora la [3], la si moltiplichi per $\Omega$ e si ponga

$$
\Omega b=\sigma \text { (curva normale del lago) }
$$


e

$$
u=\xi \Omega
$$

si ottiene:

$$
\frac{\partial^{2} u}{\partial t^{2}}=g \sigma \frac{\partial^{2} u}{\partial s^{2}}
$$

con

$$
s=\int_{0}^{x} b d x
$$

Ammesso ora che $u$ sia rappresentabile da una serie del tipo

$$
u=\sum f_{i}(s) \operatorname{sen} w_{i} t
$$

si deve avere

$$
\sigma \frac{d^{2} f_{i}}{d s^{2}}+\frac{4 \pi^{2}}{g T_{i^{2}}} f_{i}=0
$$

se poi in particolare si pone $z=\frac{s}{A} \quad(A=$ area totale del lago $) \mathrm{e}$ $\lambda=\frac{4 \pi^{2} A^{2}}{g T-}$ si ha

$$
\frac{d^{2} f}{d z^{2}} \div \frac{\lambda}{\sigma(z)} f=0
$$

cui vanno associate le condizioni ai limiti

$$
f=0 \quad \text { per } z=0, z=1
$$

L'utilizzazione della [9] può avvenire per diverse vie.

$1^{\circ}$ ) Se la funzione $\sigma(z)$ è abbastanza semplice, essa può essere integrata direttamente.

Così ad esempio se $\sigma(z)$ è del tipo $K_{0} z^{m}$, l'equazione [9] diviene

$$
\frac{d^{2} f}{d z^{2}} \div \frac{K_{1}}{z^{m}} f=0 \quad\left(K_{1}=\lambda / K_{0}\right)
$$

Essa ha come integrale generale

$$
f=\sqrt{z} Z \frac{1}{2-m}\left(\frac{\underline{2} \cdot \sqrt{\frac{K}{2}}}{2-m} \cdot z^{\frac{2-m}{m}}\right)
$$


avendo indicato con $Z$ una combinazione lineare delle funzioni $J$ e $Y$.

Le condizioni ai limiti $(\xi \Omega$ finito) consentono poi di utilizzare il solo integrale particolare $I$.

Se ad esempio il bacino in studio è a profondità e larghezza linearmente variabili, si ha

$$
\begin{aligned}
& h=h_{0} \frac{x}{a} \\
& b=b_{0} \frac{x}{a} \\
& s=\int_{0}^{x} v_{0} \frac{x}{a} d x=\frac{1}{2} \frac{b_{0}}{a} x^{2} \\
& z=\frac{1}{2} \frac{b_{0}}{a} \times \frac{2}{b_{0} a}-\frac{x^{2}}{a^{2}} \\
& \sigma=a h_{0} \frac{x}{a} b_{0}^{2} \frac{x^{2}}{a^{2}} .
\end{aligned}
$$

ove $\alpha$ dipende dalla forma della sezione trasversale del lago $(\alpha=1$ per la rettangolare, $a=1 / 2$ per la triangolare, ecc.), e quindi

$$
\sigma=\alpha h_{0} b_{0}=z^{3 / 2} .
$$

Così per un lago a sezione rettangolare $K_{1}$ risulta

$$
K_{1}=\frac{4 \pi A^{2}}{g T^{2}}=\frac{1}{h_{0} b_{0}{ }^{2}}
$$

e l'equazione determinatrice dei periodi e, essendo $m=\frac{3}{2}$,

$$
4 \sqrt{K_{1}}=\varrho_{2 i}
$$

avendo indicato con $\underline{o}_{2 i}$ gli zeri della funzione $I_{2}$, integrale particolare della [10] per il caso in esame.

Per fare un esempio numerico, se si assume

$$
h_{0}=50 \mathrm{~m}, \quad b_{0}=300 \mathrm{~m}, \quad a=1800 \mathrm{~m}
$$


poiché è

$$
\begin{aligned}
& \varrho_{21}=5,136 \\
& \varrho_{22}=8,417
\end{aligned}
$$

i corrispondenti periodi risultano

$$
\begin{aligned}
& T_{1}=\frac{4 \cdot 2 \pi \cdot A}{\varrho_{21} \sqrt{g h_{0} b_{0}}}=221 \mathrm{sec} \\
& T_{2}=\frac{4 \cdot 2 \pi \cdot A}{\varrho_{22} \sqrt{g h_{0} b_{0}^{2}}}=134 \mathrm{sec}
\end{aligned}
$$

$2^{\circ}$ ) Se la curva normale non è facilmente esprimibile, conviene ricorrere al metodo di Hidaka, ben noto e sul quale non ci soffermiamo.

Richiameremo soltanto che gli autovalori della equazione [9] ren. dono stazionario l'integrale

$$
I=\int_{0}^{1}\left[\left(\frac{\partial f}{\partial z}\right)-\frac{\lambda}{\sigma(z)} i\right\rceil d z
$$

in quanto la [9] può essere interpretata come equazione di Eulero della [12].

Il metodo di Ritz applicato alla [12], assumendo per $f$ l'espressione

$$
f=\Sigma a_{t}(1-z) z^{t}
$$

conduce allora alla determinazione degli autovalori $\lambda_{1}, \lambda_{2} \ldots$, cui corrispondono i periodi $T_{1}, T_{2} \ldots$

Nello sviluppo dei calcoli intervengono integrali del tipo [13], che vedremo nel paragrafo successivo, in genere eseguibili numericamente.

3. - Quanto detto al paragrafo 2 è stato applicato a quattro bacini artificiali, di batimetria ben nota.

Di ciascuno di essi riportiamo in allegato la curva normale, l'andamento delle profondità e della larghezza in superficie, (Figg. 2,3,4,5) mentre le caratteristiche principali sono riportate nella Tabella I. 

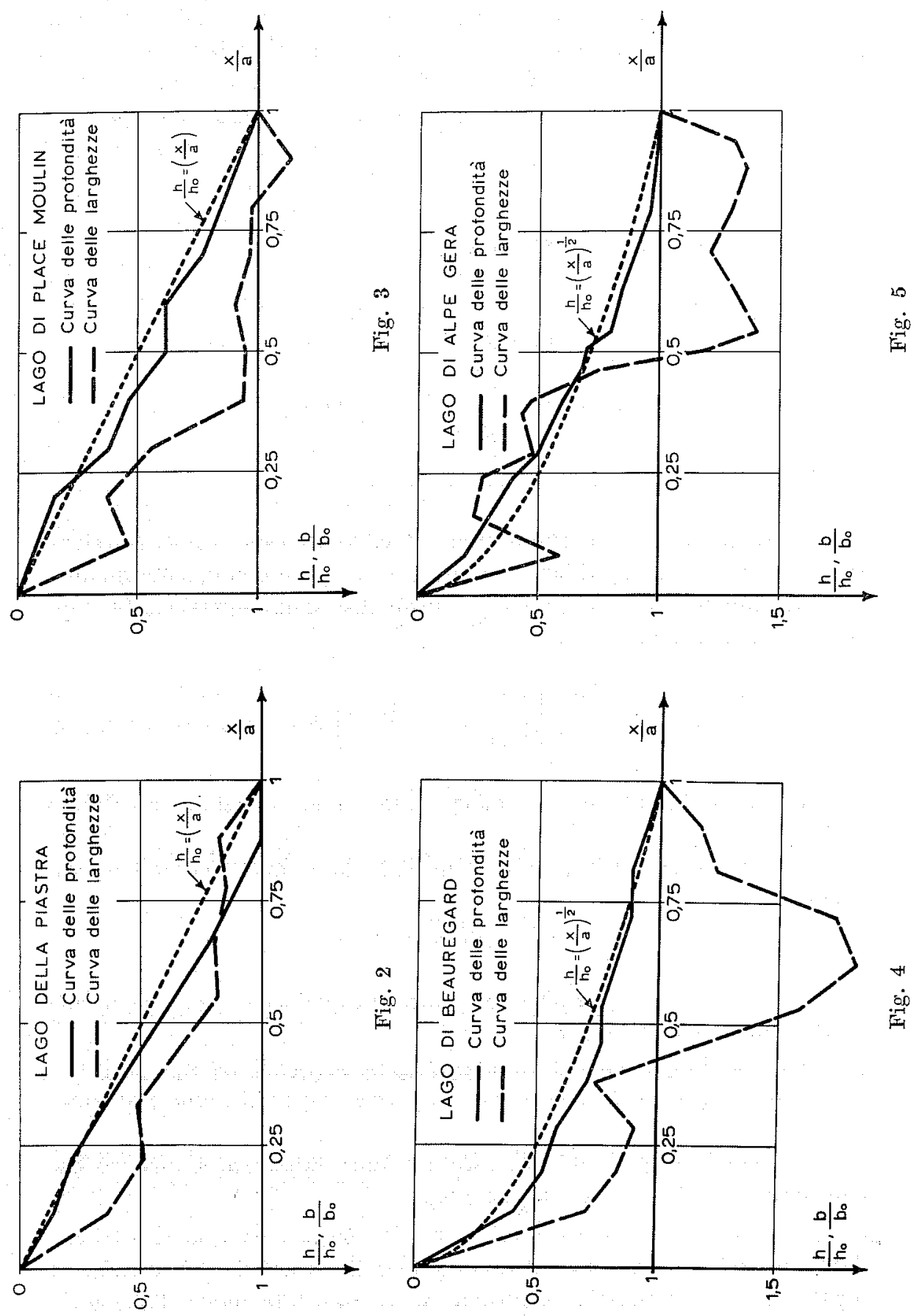
CARATTERISTICIE IDRODINAMICHE DI ALCUNI LAGHI ARTIFICIALI 399

Tabella I

\begin{tabular}{|c|c|c|c|c|}
\hline Isago & $\begin{array}{l}h_{0} \\
\mathrm{~m}\end{array}$ & $\begin{array}{l}b_{0} \\
\mathrm{~m}\end{array}$ & $\underset{\mathrm{m}}{a}$ & $\frac{1}{m^{2}}$ \\
\hline Piastra. & 56 & 406 & 1800 & $4,59 \cdot 10^{5}$ \\
\hline Place Moulin . . & 130 & 501 & 4000 & $1,63 \cdot 10^{6}$ \\
\hline Beauregard . . . & 85 & 316 & 4320 & $1,49 \cdot 10^{5}$ \\
\hline Alpe Gera. & 100 & 500 & 2450 & $1,08 \cdot 10^{6}$ \\
\hline
\end{tabular}

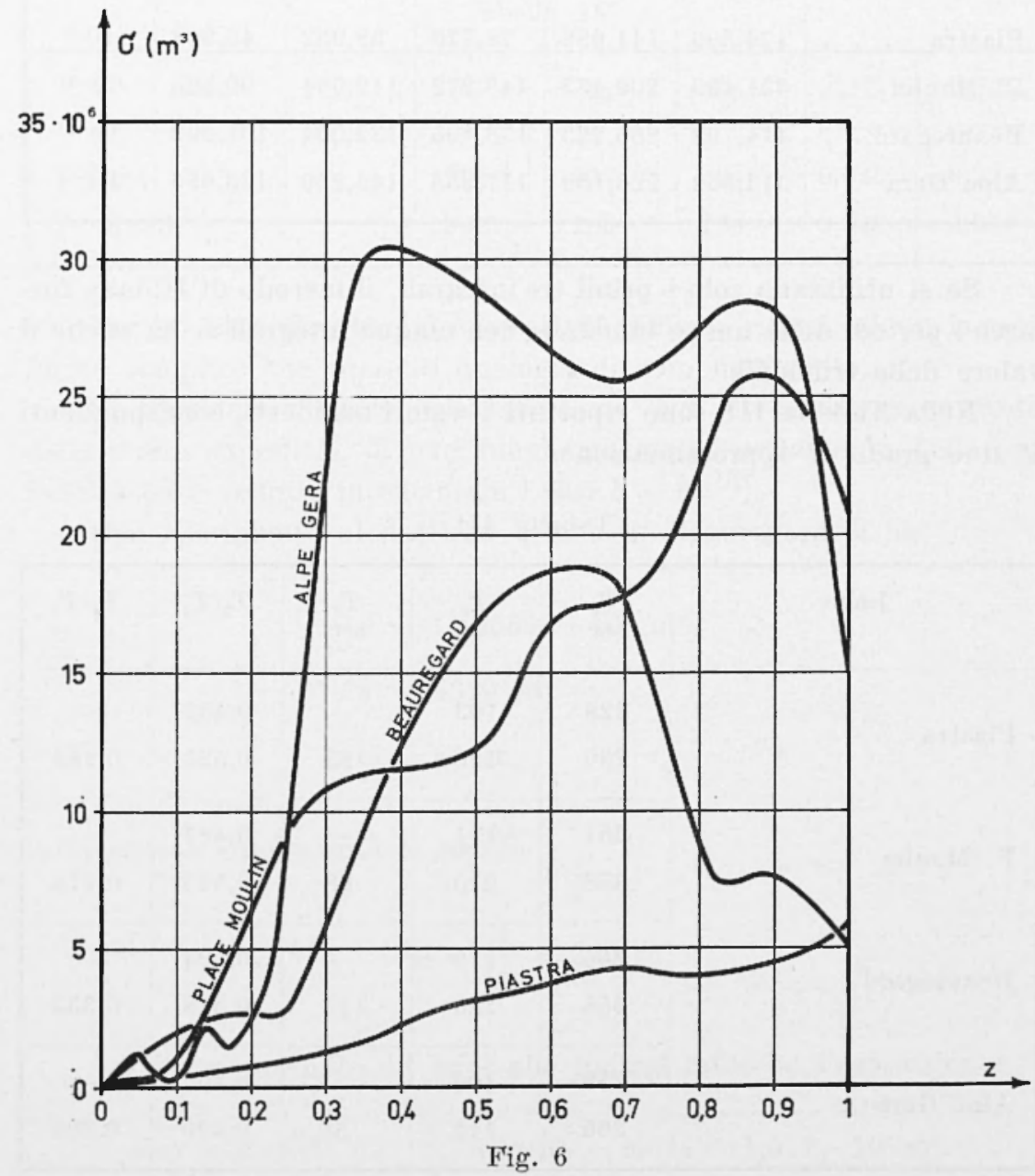


Nella Tabella II abbiamo riportato i valori degli integrali

$$
I_{n}=\int_{0}^{1} \frac{(1-z)^{2} z^{n+2}}{\sigma(z)} d z
$$

calcolati numericamente, non essendo stato agevole esprimere la $\sigma(z)$ per via analitica.

Tabella II

\begin{tabular}{|c|c|c|c|c|c|c|}
\hline Lago & $I_{0}$ & $I_{1}$ & $I_{2}$ & $I_{3}$ & $I_{4}$ & \\
\hline Piastra & 174,509 & 111,688 & 78,779 & 58,952 & 45,903 & $10^{-8}$ \\
\hline P. Moulin & 331,490 & 209,433 & 143,272 & 112,954 & 90,425 & $10^{-11}$ \\
\hline Beauregard & 414,792 & 256,223 & 178,495 & 132,054 & 101,292 & $10^{-11}$ \\
\hline Alpe Gera & 313,552 & 226,159 & 177,955 & 146,209 & 123,055 & $10^{-11}$ \\
\hline
\end{tabular}

Se si utilizzano solo i primi tre integrali, il metodo di Hidaka fornisce i periodi della uni- e binodale; con cinque integrali si ha anche il valore della trinodale.

Nella Tabella III sono riportati i valori anzidetti, corrispondenti ai due gradi di approssimazione.

Tabella III

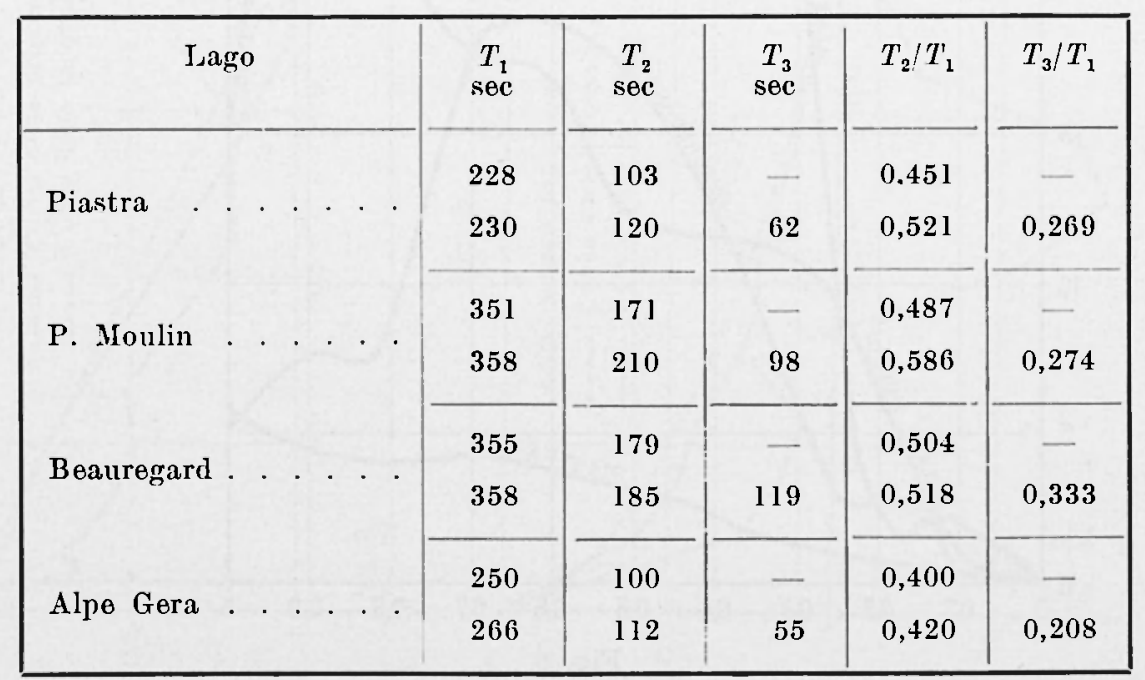


4. - In relazione al particolare andamento della curva normale dei laghi della Piastra e Place Moulin, e all'andamento abbastanza regolare delle profondità, abbiamo fatto un confronto tra $\mathrm{i}$ periodi trovati e quelli di un lago con andamento delle profondità lineare da $h_{0}$ a 0 e larghezza costante. Un lago siffatto presenta ovviamente una curva normale lineare.

Per un lago di questo tipo abbiamo visto che la relazione determinatrice dei periodi è la [7] tramite la quale abbiamo calcolato i valori riportati nella Tabella IV e che sono in buon accordo con quelli della Tabella III, almeno per quanto riguarda i periodi delle uni- e binodali.

Tabella IV

\begin{tabular}{|c|c|c|c|c|c|}
\hline I aago & $T_{1}$ & $T_{2}$ & $T_{3}$ & $T_{2} / T_{1}$ & $T_{3} / T_{1}$ \\
\hline Piastra & 251 & 137 & 94 & 0,545 & 0,376 \\
\hline P. Moulin & 367 & 200 & 138 & 0,545 & 0,376 \\
\hline
\end{tabular}

Per gli altri due bacini è assai più difficile rifarsi ad un lago di forma semplice che presenti analogia di comportamento.

Si consideri a titolo orientativo un lago di larghezza costante, della stessa superficie, di pari lunghezza e pari andamento delle profondità (si è assunto in entrambi i casi $h=c x^{1 / 2}$ ).

Con riferimento ai dati del bacino di Beauregard si ha

$$
\begin{aligned}
& A=1,495520 \cdot 10^{6} \mathrm{~m}^{2} \\
& a=4320 \mathrm{~m} \\
& h_{0}=85 \mathrm{~m},
\end{aligned}
$$

la larghezza equivalente $b_{0}$ risulta

$$
b_{0}=\frac{\Delta}{a}=346 \mathrm{~m}
$$

La curva normale, in base alle ipotesi fatte ha l'espressione:

$$
\sigma(z)=K_{0} z^{1 / 2} \quad \operatorname{con} K_{0}=b_{0}{ }^{2} h_{0}=1,017 \cdot 10^{7} \mathrm{~m}^{3} .
$$


Dalla [11] si deduce l'equazione risolvente dei periodi $\left(m=\frac{1}{2}\right)$

$$
-\frac{4}{3} \sqrt{K_{1}}=\varrho_{2 / 3, i}
$$

ovvero

$$
\frac{ \pm}{3} \frac{2 \pi A}{\sqrt{g} \overline{h_{0} b_{0}^{2}}}-\frac{1}{T_{i}}=\hat{-}_{2 / 3 .}
$$

poiché gli zeri della funzione $\vec{J}_{z_{i}}$ valgono

$$
\begin{aligned}
& \varrho_{2 / 3, \imath}=3,3750 \\
& \varrho_{\frac{I_{-},:}{}}=6,5297
\end{aligned}
$$

i corrispondenti periodi risultano

$$
\begin{aligned}
& T_{1}=371 \mathrm{sec} \\
& T_{2}=191 \mathrm{sec}
\end{aligned}
$$

con un rapporto $T_{2} / T_{1}$ pari a 0,516 e in buon accordo con i dati della Tabella III.

Lo stesso criterio, applicato al lago di Alpe Gera, e sempre nella ipotesi di curva delle profondità di andamento

$$
h=c x^{1 / 2}
$$

ha portato a valori invece assai diversi rispetto a quelli della Tabella III e precisamente

$$
\begin{aligned}
& T_{1}=194 \mathrm{sec} \\
& T_{2}=100 \mathrm{sec} .
\end{aligned}
$$

Tale discordanza era peraltro prevedibile a priori, in quanto col metodo di Hidaka si era ottenuto $T_{2} / T_{1}=0,42$ (v. Tab. III) contro il valore 0,516 cui ci avrebbe condotto un calcolo analogo a quello fatto per il bacino di Beauregard.

Non è quindi agevole riportarsi a laghi di forma semplice che oscillino con periodo analoghi a quelli di bacini naturali. 
Qualora questo fosse possibile, come per il caso del lago di Beauregard, resterebbero poi in genere grosse discordanze nella posizione delle linee nodali, sicché sembra più logico procedere come indicato al paragrafo seguente.

丂. - Un altro modo di affrontare il problema, può essere quello di integrare direttamente la [9] scrivendola alle differenze finite

$$
f_{n+2}=2 f_{n+1}-f_{n}-\frac{\lambda}{v(z)}(\Delta z)^{2} f_{n+1}
$$

suddividendo l'intervallo $0 \div 1$ di variazione della $z$ in un numero conveniente di passi.

In una prima fase possono essere ricercati gli autovalori $\lambda$ controllando che sia $f=0$ per $z=1$; successivamente tenuto presente che la sopraelevazione massima in ogni sezione risulta, per la [2],

$$
\eta=-\frac{d f}{d z} \cdot \frac{1}{A}
$$

dall'andamento di $\frac{d f}{-\frac{d}{-} z}$ si può desumere la posizione delle linee nodali.

Il calcolo è stato condotto con un elaboratore elettronico HewlettPackard e relativo plotter, assumendo nell'intervallo $0 \div 1$ un passo di integrazione pari a $1 / 1000$.

I periodi ottenuti, riportati nella Tabella $\mathrm{V}$, mostrano una buona concordanza con quelli della Tabella III, che discendono dall'applicazione di quanto indicato al paragrafo 2 , punto $2^{\circ}$ ); gli scarti divengono però assai sensibili per le trinodali, presumibilmente per una lenta convergenza del metodo di Hidaka.

\section{Tabella V}

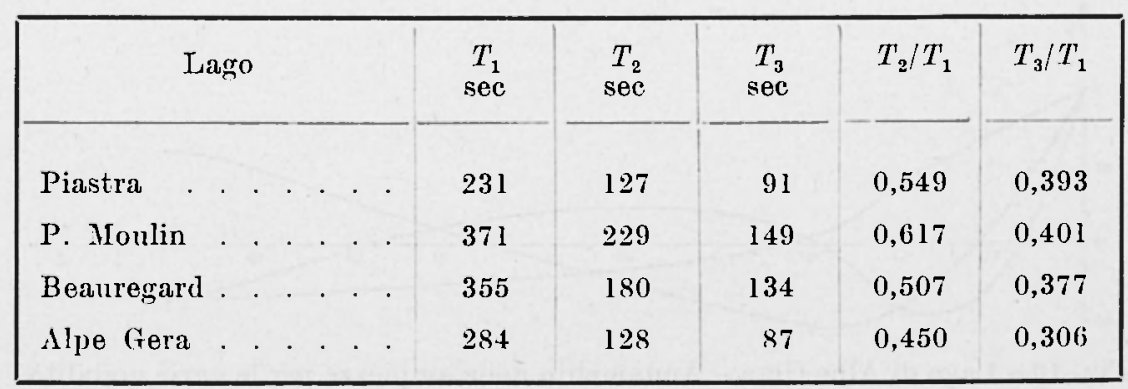




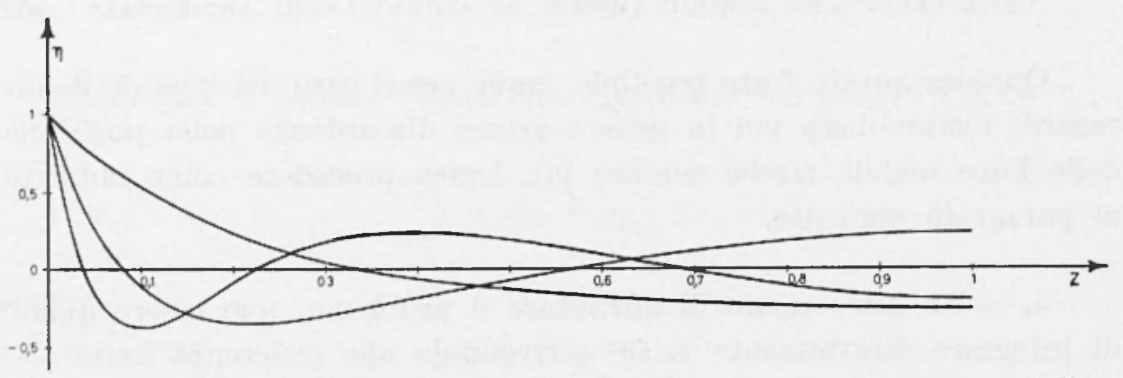

Fig. 7 - Lago della Piastra - Andamento delle ampiezze per le varie nodalità.

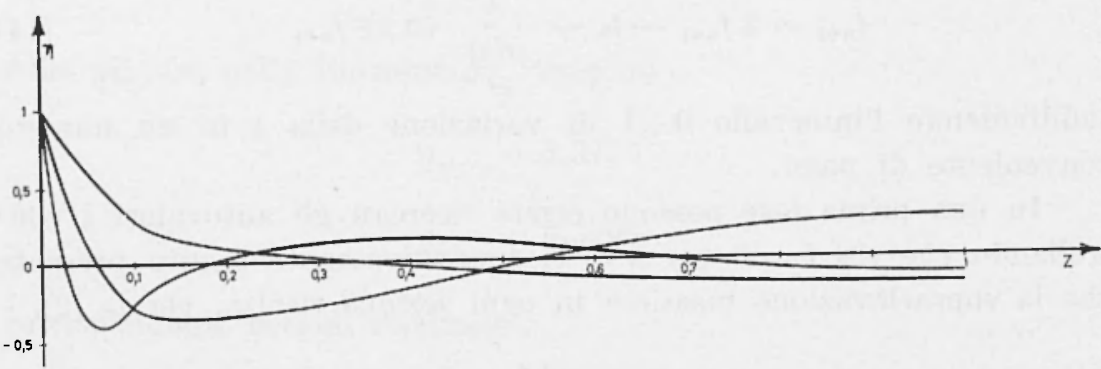

Fig. 8 - Lago di Place Moulin - Andamento delle ampiezze per le varie nodaliti.

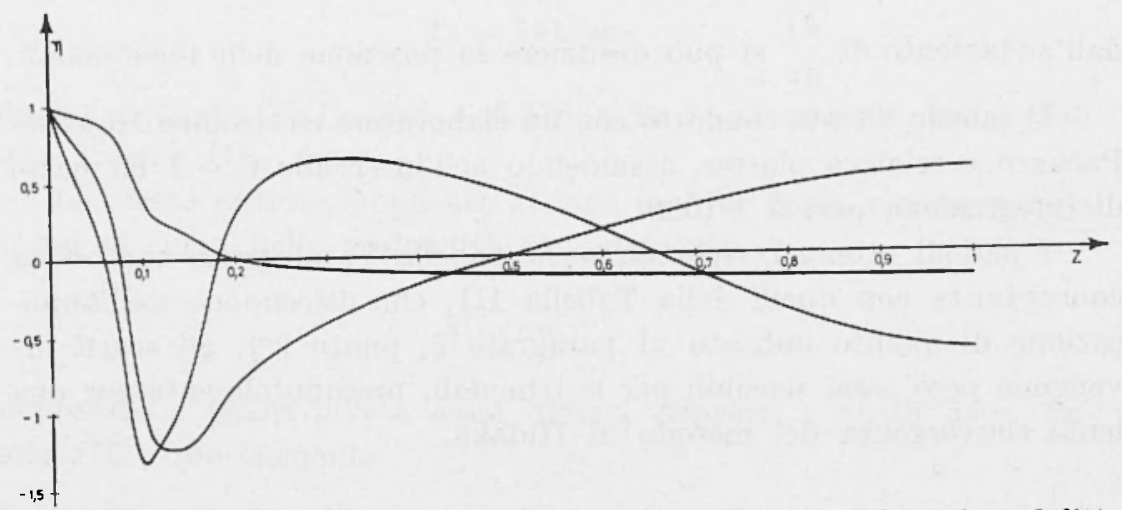

Fig. 9 - Lago di Beauregard - Andamento delle ampiezze per le varie nodalità.

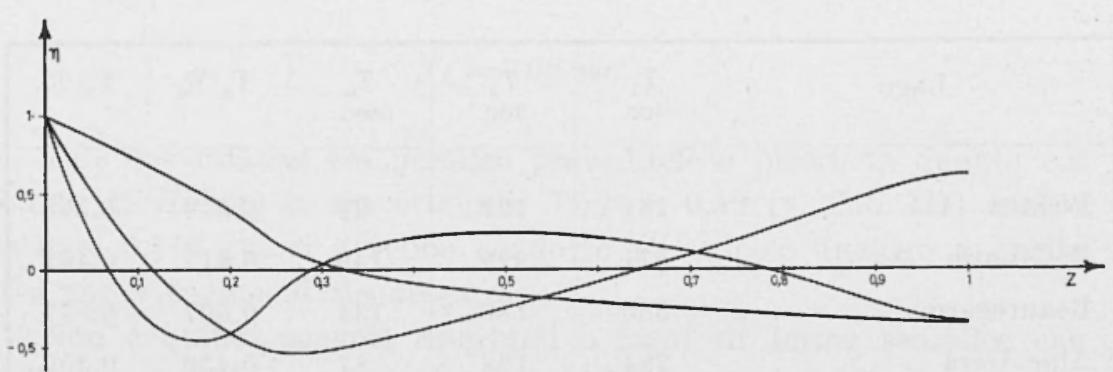

Fig. 10 - Lago di Alpe Gera - Andamento delle ampiezze per le varie nodalità. 
Per i laghi della Piastra e P. Moulin l'accordo è migliore (anche per le trinodali) con i dati della Tabella IV, ottenuti approssimando l'effettiva curva normale dei due laghi ad una retta.

La conferma qualitativa la si è avuta dall'andamento delle ampiezze che risulta molto simile a quello di un lago con $\sigma(z)$ rettilinea.

Nello svolgimento dei calcoli si è poi riscontrata la necessità, qualora la curva normale presenti in alcuni tratti ordinate assai modeste (caso del bacino di $\mathrm{Alpe}$ Gera) di raffittire notevolmente il passo di integrazione.

L'andamento delle ampiezze per le uninodali, binodali e trinodali è riportato in nelle figure $78,9,10$.

\section{BIBIIOGRAFIA}

(1) Bovasse H., 1924. - Houlle, Rides, Seiches el Marees. Parigi.

$\left({ }^{2}\right)$ Caloi P., Romundid G., Spadea M. C., 1967. - Caratteristiche idrodinamiche del lago di Sauris. "Annali di Geofisica", $\mathbf{X X}, 3$.

(3) Chrtstat. G., 1905-1906-1907/8. - On the Hydrodinamical theory of seiches. "Transaction of the Royal Society of Edimburg ", XLIV, XLV, XLVI.

(4) HidAкA K., 1936. - Applications of Ritz's Variation Method to the determination of seiches in a lake. The memoirs of the "Imperial Marine Observatory ", VI. 2.

(5) Romunidi G., Spadsa M. C.. 1966. - Studio preliminare sulle oscillazioni libere del lago di Sauris a due diverse quote. "Annali di Geofisica", XIX, 4 . 